\title{
Quaderni
}

QUADERNI Communication, technologies, pouvoir

89 | Hiver 2015-2016

Penser l'avenir : le CESTA, un think tank atypique

\section{Le GRET, une histoire parallèle à celle du CESTA dans le domaine de la coopération internationale : un témoignage}

Marc Lévy

\section{(2) OpenEdition Journals \\ Édition électronique \\ URL : http://journals.openedition.org/quaderni/958 \\ DOI : 10.4000 /quaderni.958 \\ ISSN : 2105-2956 \\ Éditeur \\ Les éditions de la Maison des sciences de l'Homme}

Édition imprimée

Date de publication : 5 janvier 2016

Pagination : 65-74

Référence électronique

Marc Lévy, « Le GRET, une histoire parallèle à celle du CESTA dans le domaine de la coopération internationale : un témoignage », Quaderni [En ligne], 89 | Hiver 2015-2016, mis en ligne le 05 janvier 2018, consulté le 10 décembre 2020. URL : http://journals.openedition.org/quaderni/958 ; DOI https://doi.org/10.4000/quaderni.958 


\section{Dossier}

\section{Le GRET,}

Vers le milieu des années 70 deux anciens " coopérants techniques » vont jeter les bases une histoire d'une aventure incarnée dans une organisation qui dure encore aujourd'hui et qui, sans être directement liée à celle du CESTA, n'en est pas parallèle à celle du CESTA dans le domaine de la coopération moins proche par certaines de ses ambitions, sans compter l'effet d'époque dont elles sont toutes les deux le reflet intéressant à considérer. Il faut ajouter qu'ayant été partie prenante de cette aventure et étant un ami d'Yves Stourdzé, nous avons eu l'occasion de confronter nos situations et d'en discuter au point de certainement créer un lien entre ces deux aventures, lien que j'espère mettre en évidence pour rendre hommage à son influence.

\section{internationale :} un témoignage
Directeur de la prospective
GRET

Directeur de la prospective
GRET

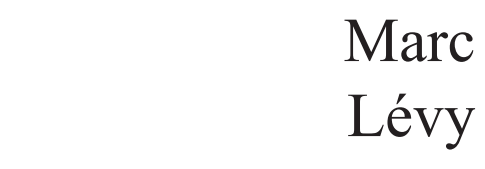

Les deux agronomes en question revenaient l'un d'Afrique francophone, l'autre d'Amérique centrale en ayant éprouvé chacun à leur façon l'inadaptation des modèles techniques appris à l'école d'agronomie aux situations auxquelles la coopération les confrontait. Après tout ils n'avaient pas été formés a priori pour agir en milieu tropical. Ce qui était en question n'était pas tant leur formation que le fait d'envoyer de jeunes ingénieurs, dans le cadre de leur service militaire, exercer leur fraîches compétences avec si peu de préparation dans des réalités quelque peu décalées. Comme le dispositif français de coopération avec les pays en développement (PED) était à l'époque très conséquent, avec une administration spécialisée importante, des instituts de recherche entièrement consacrés aux PED, des bureaux d'étude, des milliers de « coopérants techniques », les deux agronomes vont revenir fortement animés d'un point de vue critique sur les modèles techniques, les méthodes de recherche, le fonctionnement administratif, 
autrement dit plus largement, sur la politique française de coopération et la vision des rapports « Nord / Sud » qu'elle reflétait et servait.

Ils vont s'intéresser au courant des « technologies appropriées » qui commençait à essaimer dans le monde à partir du Royaume-Uni avec les travaux de Schumacher (« Small is beautiful») et la création de 1'ITDG (Intermediate Technology Development Group), avec l'idée de créer la branche française de ce courant. Ce sera le GRET (Groupe de Recherche et d'Échange Technologique) initié en 1974 et officiellement créé sous forme associative en 1976. Bien qu'abordées différemment, nous convergions avec Yves Stourdzé sur l'importance du paramètre technologique dans les problématiques de développement et sur l'intérêt de l'aborder avec d'autres savoirs que les sciences de l'ingénieur pour traiter des rapports entre techniques et sociétés. Cette convergence, exprimée dans nos conversations, eut un effet stimulant et consolidant, dans un environnement pas toujours perméable.

\section{Innovation et institution}

En plus de la technologie comme sujet d'intérêt commun, nous avions tous les deux des difficultés à propos du positionnement institutionnel (à la fois « dedans » et « dehors » par rapport à l'administration) et une volonté d'innover. Une parenthèse institutionnelle s'impose donc car tout à fait essentielle pour situer une des originalités de l'initiative et illustrer les jeux de pouvoir à l'œuvre.

À l'époque, au milieu des années 70, du point de vue administratif et politique, le dispositif de coopération (hors les questions de défense) reposait principalement : sur trois ministères ${ }^{1}$ (Coopération, Relations extérieures, Économie et Finances), plus une institution financière (la Caisse Centrale de Coopération Économique CCCE) et la présidence de la République (Cellule Afrique de l'Élysée). Ce dispositif reflétait encore fortement les décisions prises au lendemain des indépendances, la « coopération » ayant été inventée pour maintenir une certaine influence sur les anciennes colonies. D'où le partage des tâches au sein d'un ensemble dont on comprend tout de suite que le côté éclaté ne sera pas le moindre de ses problèmes : (1) l'Élysée s'occupait du " politique », (2) le ministère de la Coopération s'occupait principalement de la coopération technique avec les pays d'Afrique francophone ; (3) le ministère des Relations extérieures avait en charge les actions de coopération avec le reste du monde (développé et en développement) ; (4) le ministère de l'Économie et des Finances régissait les contributions financières, la zone franc et la représentation dans les instances multilatérales ; (5) la CCCE finançait les infrastructures.

L'analyse critique de ce dispositif, devenu progressivement obsolète et difficile à réformer, est une histoire passionnante, suffisamment bien documentée ${ }^{2}$ pour ne pas avoir à le faire dans le cadre de cet article. Il fallait seulement situer la différence, du point de vue des ministères concernés, entre d'une part le « cœur » du dispositif, le ministère de la Coopération qui mobilisait une grosse part des moyens humains et financiers et représentait une certaine permanence de la « France d'outre-mer » malgré l'arrivée de professionnels (agronomes, enseignants, médecins, etc.) motivés par le « développement » et d'autre 
part celui des Relations extérieures, couvrant un vaste domaine (géographique et sectoriel) où la coopération technique avec les PED était très marginale comparativement aux relations culturelles et scientifiques véhiculant l'excellence et l'influence françaises.

C'est justement dans la « marge » que l'innovation va pouvoir avoir lieu. Un de nos deux agronomes va avoir la possibilité à son retour de coopération, d'être recruté au ministère des Relations extérieures pour s'occuper de la coopération technique agricole avec les PED autres que ceux d'Afrique francophone (Amérique latine et centrale, Caraïbes, Asie, Maghreb, Afrique anglophone).

Il va créer un « Service d'appui technique », pour mieux former les coopérants et leur apporter un suivi, service qu'il placera en dehors du ministère pour lui donner des marges de manœuvre et dont il confiera la direction au « deuxième » agronome. Ils créeront et installeront le GRET au sein de ce Service d'appui technique pour avoir un outil, à la fois indépendant et articulé à la politique de coopération qu'il s'agit de faire évoluer en jouant sur l'interne et l'externe. Et la hiérarchie du ministère, du moins une partie, apportera son appui, en partie pas fâchée d'être un lieu d'innovation face au ministère de la Coopération. Quand on sait que finalement en 1998, vingt ans plus tard, le ministère de la Coopération disparaitra pour être fusionné au sein du ministère, devenu des Affaires étrangères, on peut se faire une idée des rapports de force institutionnels à l'œuvre, auxquels des aventures atypiques, comme celle du GRET et d'autres, ont apporté leur contribution. La question étant de savoir dans quelle mesure il s'agit d'une contribution de « rupture » ou « d'adaptation »?

En tout cas, cette situation institutionnelle, en faisant travailler le GRET au Nicaragua, en Haïti, en Iran, en Inde, en Éthiopie, au Mexique, au Brésil, etc., nous a fait acquérir une vision du monde plus ouverte que « la France-Afrique », vision qui nous a fait percevoir comment la mondialisation allait perturber la notion de « Tiers-Monde » et l'opposition « Nord / Sud ».

D'une part avec l'apparition des « pays émergents » sortis en fait du sous-développement au cours du $\mathrm{XX}^{\mathrm{e}}$ siècle, d'autre part avec les réalités de la pauvreté, complexifiées par celles des inégalités, qui ont accompagné la progression de la mondialisation et de la globalisation (capitalisme financier et économie néolibérale). La pauvreté et les inégalités ont bousculé les frontières géographiques pour devenir plus fortes au sein des pays qu'entre les pays. Difficile de voir le monde aujourd'hui divisé entre un « Nord» riche et un « Sud » pauvre, il y a d'une part des pauvres au Nord et des riches au Sud et d'autre part des « pays à revenus intermédiaires » qui sont venus bousculer cette dichotomie. Pour autant, le monde n'est pas devenu homogène même si des « classes moyennes » existent maintenant partout, mais peut-être davantage interdépendant. En matière de coopération, le panorama des acteurs a progressivement considérablement changé : le Brésil, la Chine, les pays arabes sont devenus des « bailleurs »; des fondations privées mobilisent plus d'argent que l'aide publique ; des ONG sont devenues des « acteurs globaux», certaines ayant une puissance multinationale au même titre que les firmes, etc. Mais avons-nous réussi 
à percevoir, à faire valoir et même à intégrer ces « ruptures »?

\section{Le lien avec la coopération scientifique}

Avant de répondre à la question, il faut faire un détour par le monde de la recherche. Les technologies appropriées, tout en étant des alternatives à celles qui symbolisaient le modèle intensif occidental, demeuraient classiques dans le champ de l'innovation en restant dans le domaine de la technique : la traction attelée avec les animaux contre le tracteur, la pompe solaire contre le groupe électrogène, les briques de terre compactée contre le ciment, etc. En retournant les termes, donc en parlant d'appropriation technologique, l'ambition était d'introduire les acteurs, de parler d'innovation en termes d'usage, de faire le lien avec les processus productifs, et par conséquent de traiter des rapports entre techniques et sociétés, avec le recours aux sciences humaines. Les ingénieurs que nous étions se sont mis progressivement à s'intéresser aux systèmes, aux groupes sociaux, aux institutions, à l'économie, à l'économie politique même, aux rapports de pouvoir, etc. Et parce que, dans les sphères dirigeantes, les technologies appropriées faisaient plutôt sourire tandis que l'appropriation manquait de crédibilité méthodologique, nous avons cherché à consolider nos approches et à les faire reconnaître par le milieu de la recherche. Nous ne parlions pas de $«$ Technologie, Croissance, Emplois $»^{3}$, notre vocabulaire n'était pas le même que celui du CESTA, mais la proximité des questionnements était pour moi évidente et encourageante.

Dès le début de l'aventure du GRET, le lien avec les « coopérants techniques ${ }^{4}$ » avait confirmé qu'ils pâtissaient d'un certain isolement, manquant de relations entre eux et de base arrière pour avoir accès à des sources d'informations. En particulier, les résultats scientifiques circulaient peu, le lien entre la recherche et le développement n'était pas une préoccupation dominante. Le GRET a d'emblée construit son utilité sociale autour d'une activité de « communication » mettant en circulation, avec des outils « appropriés », les informations nécessaires aux développeurs. Comparativement, cette activité mettait en évidence combien le monde de la recherche était peu sensibilisé à ce qui allait devenir un sujet en tant que tel : l'information et la culture scientifique et technique.

C'est avec ces deux sujets, l'appropriation comme méthodologie d'innovation et l'information comme outil de communication de la recherche avec le développement, que nous avons abordé, à la fin des années 70, forts de l'appui que nous recevions du ministère des Relations extérieures, le secrétariat d'État à la Recherche, pour faire valoir notre existence et susciter un intérêt.

Ce fut une bonne anticipation. Quand le candidat socialiste a gagné les élections présidentielles en 1981 et pris toute une série de décisions novatrices, nous avons fait partie des bénéficiaires : le président du GRET est entré au Cabinet du ministre de la Coopération, il m'a été confié la présidence de la commission du Programme mobilisateur sur l'information scientifique et technique au service du développement, le GRET a été chargé d'animer les réseaux mis en place pour rapprocher la recherche et le développement et des postes de chercheurs nous ont été attribués pour consolider cette fonction d'interface et notre 
existence dans le dispositif de coopération.

Dans une moindre mesure que le CESTA, nous avons donc connu dans ces années 80 le passage de la marge à la lumière. En même temps, nous avions de quoi échanger car nous avions fait, eux comme nous, au cours de cette ascendance, l'expérience de l'intensité des rapports de force.

Nos idées indéniablement progressaient, mais l'adversité et la concurrence également. Dans notre cas, elles nous avaient fait quitter le giron protecteur du ministère des Relations extérieures suite à la fusion, qui n'avait pas voulu de nous, du Service d'appui technique (au sein duquel, rappelons-le, nous étions installés) avec la structure équivalente du ministère de la Coopération. Nous avons assumé notre statut d'association autonome en déménageant pour nous installer dans des locaux indépendants. Heureusement l'évolution du côté de la recherche agronomique ${ }^{5}$ allait nous ouvrir une nouvelle porte.

Cette évolution est à l'image des réalités internationales, mais aussi des réalités institutionnelles françaises ${ }^{6}$. Après les « indépendances », la France, contrairement aux autres puissances coloniales qui se retireront des dispositifs de recherche dans les pays nouvellement indépendants, maintiendra un partenariat en recherche agronomique. Cette recherche était menée à la fois, dans un contexte concurrentiel pas toujours bénéfique, au sein d'un office, héritier de l'ancien Office de la Recherche Scientifique Coloniale, devenu en 1953, l'Office de Recherche Scientifique et Technique d'Outre-mer (ORSTOM et plus tard, Institut de Recherche pour le Développement - IRD). Et par ailleurs, la recherche était menée au sein de huit instituts spécialisés (café, cacao, coton, caoutchouc, fruits, bois, etc.). Dans les années 70 ce dispositif a eu du mal à se financer et se voyait concurrencer par de nouveaux grands centres internationaux de recherche agronomique ${ }^{7}$ soutenus par les fondations américaines (Rockefeller en particulier) et la Banque mondiale au nom de la « Révolution verte » et de la volonté de dominer ${ }^{8}$. Moyennant quoi les instituts furent réunis en 1970 au sein du GERDAT (Groupement d'Études et de Recherche pour le Développement de l'Agronomie Tropicale) qui bénéficia en 1973 de la construction à Montpellier d'un nouveau complexe. Il fallait ce détour pour pouvoir situer le débat de la fin des années 70 .

Le ministère de la Coopération voulait créer un «pôle tropical » avec le GERDAT et l'ORSTOM, sous l'égide de ce dernier. Opposés à ce schéma et à cette domination, d'aucuns vont construire une alternative sous la forme d'un « pôle agronomique » fruit du rapprochement du GERDAT avec l'Institut national de la recherche agricole (INRA). Et « en embuscade », certains services du ministère des Finances militaient pour la disparition de la recherche agronomique tropicale française, trop coûteuse et atypique à l'échelle mondiale, au profit d'une participation au système international.

Le ministre de la Coopération s'oppose à la seconde solution, mais jusqu'en mai 81 la fusion GERDAT/ORSTOM n'arrive pas à se faire. La gauche, arrivée au pouvoir, va annuler ce projet qualifié de " ghetto tropical »; le ministère des Finances s'opposera au rapprochement avec l'INRA qui supposait de créer des postes de fonctionnaires. Bilan, après les Assises de la 
Recherche, qui ont relancé la dynamique scientifique (une Loi, un ministre d'État, des recrutements, un budget en augmentation), la décision fut prise de fusionner les instituts et de transformer le GERDAT en EPIC (Établissement public à caractère industriel et commercial). Le nouveau ministre de la Recherche a fait accélérer le processus, en un an fut créé, fin 1984, le CIRAD (Centre de Coopération Internationale en Recherche Agronomique pour le Développement), qui voyait arriver à la direction, sa « cheville ouvrière », l'administrateur du GERDAT opposé à la fusion avec l'ORSTOM ; à la direction scientifique un acteur issu de la société civile porteur d'une vision renouvelée de la recherche et à la présidence, le président de l'INRA' !

Le directeur général et le directeur scientifique étaient très favorables aux thématiques que nous portions également : la recherche-développement, les approches systèmes, les exploitations familiales et les technologies alternatives, la communication scientifique et technique, les alliances entre recherche et développement en particulier avec des organisations non gouvernementales comme la nôtre. Ils vont accepter de prendre en charge les postes de chercheurs accordés au GRET par le ministère de la Recherche et cofinancés par le ministère des Relations extérieures (postes qu'en tant qu'association le groupe ne pouvait pas accueillir) en agréant de les mettre ensuite à disposition de l'association dans le cadre d'une convention qui scellait une alliance sur le long terme. C'était une belle innovation, soutenue par des « parrains » courageux car il y avait des adversaires, une innovation qui confortait notre idée de faire évoluer la sphère publique de l'extérieur par des effets de levier jouant sur le «dedans-dehors » et des complicités de personnes. Trente ans plus tard, la collaboration avec le CIRAD a connu différentes phases mais la convention est toujours en vigueur.

\section{Aujourd'hui}

Que sont devenues ces innovations? Il s'est passé énormément de choses, en France, en Europe, dans le monde.

La France a connu une réforme de son dispositif de coopération étalée sur plusieurs années (1998/2004/2008) qui s'était révélée impossible à réaliser jusqu'alors. Au nom d'une vision actualisée de la mondialisation, le ministère de la Coopération, ou «ministère de l'Afrique », a disparu au profit d'une grande direction de la coopération internationale fusionnant les deux mots-clés, « Solidarité et Influence », au sein du ministère des Affaires étrangères, direction réformée par la suite en devenant la « direction de la mondialisation et des partenariats ». Il s'agissait, plutôt à juste titre, d'intégrer la coopération au développement dans la politique étrangère, mais parfois on se demande si l'intégration n'est pas synonyme de marginalisation. Et le processus de réforme n'est pas terminé puisqu'est actuellement engagée une réflexion sur « le ministère des Affaires étrangères du $\mathrm{XXI}^{\mathrm{e}}$ siècle ».

A priori, d'une certaine façon, cette réforme était une bonne nouvelle. Mais dans les faits, la réforme n'a pas vraiment modernisé notre politique de coopération, dans son contenu. Fondamentalement polycéphale, elle reste tiraillée entre des tendances contradictoires (influence ou 
solidarité, bilatérale ou multilatérale, économique ou sociale, politique ou militaire, africaine ou asiatique, etc.) qui certes, s'accommodent en coexistant, mais en faisant perdurer des rapports de force pesants, faute de faire l'objet des arbitrages nécessaires.

L'instance de coordination interministérielle (le CICID) placée sous l'autorité du Premier ministre qui en est théoriquement chargée n'est pas vraiment à l'œuvre. La distinction entre une fonction « opérationnelle » (confiée à l'Agence Française de Développement) et une fonction «politique » (que devait assurer la grande direction du ministère des Affaires Étrangères) a été rendue difficile par la faiblesse politique de cette direction (à la fois en interne au sein du ministère des Affaires Étrangères et en externe compte-tenu du poids du ministère des Finances).

De leur côté, le CIRAD et l'IRD (ex-ORSTOM) existent toujours, indépendants l'un de l'autre, sous la tutelle complexe du secrétariat d'État à l'Enseignement supérieur et à la Recherche et du ministère des Affaires Étrangères, supervisée par celui des Finances, avec des questionnements persistants, voire grandissants, sur l'intérêt, la spécificité et le modèle économique d'une « recherche pour le développement ». Laquelle recherche doit s'aligner sur des critères d'excellence scientifique tout en maintenant des relations partenariales difficiles, en particulier avec les pays du continent africain où les dispositifs de recherche ne sont pas en bonne santé et où persiste, malgré des taux de croissance positifs grâce au commerce des matières premières, une pauvreté absolue importante à cause d'une forte croissance démographique qui réduit les effets de la baisse de la pauvreté relative.

D’une façon générale, dans le monde, la pauvreté a été réduite ${ }^{10}$, grâce aux résultats obtenus en Chine et en Inde qui n'ont pourtant pas suivi les modèles libéraux prônés par la Banque mondiale et le FMI. Mais la majorité des pauvres se trouve maintenant dans les pays émergents, non bénéficiaires de « l'aide internationale » qui a pourtant pour objectif prioritaire de lutter contre la pauvreté et qui ne peut pas le faire là où elle est la plus importante. Il reste le continent africain qui voit une baisse encourageante de la pauvreté en termes relatifs mais où malgré tout le nombre de pauvres augmente compte tenu de la croissance démographique. Quant à la croissance que ces pays ont connue (y compris ceux du continent africain), elle s'est accompagnée d'une montée des inégalités, facteur d'instabilité de plus en plus évident. Le « développement » continue d'être une équation difficile entre les objectifs de croissance économique, d'équité sociale et d'équilibres environnementaux, et sans que le monde soit devenu homogène (la moitié de la richesse mondiale est détenue par $1 \%$ de la population), cette difficile équation ne concerne pas que les pays en développement. Les nouveaux Objectifs du Développement Durable ${ }^{11}$ sont devenus « universels », on parle d'avenir commun et un certain consensus s'exprime en faveur d'une « croissance inclusive et durable ». La directrice du FMI a même déclaré que «trop d'inégalités nuit à la croissance » et le Secrétaire Général de l'OCDE : "Inequality in OECD countries is at his highest since records began $\gg .{ }^{12}$

Donc d'une certaine façon les idées alternatives ont fait leur chemin, les excès inégalitaires et en- 
vironnementaux du modèle libéral font partie des problèmes contemporains et on voit progresser la nécessité de limiter les pratiques d'optimisation fiscale et d'engager une transition énergétique. Avec toutefois la même question : changement de système (rupture) ou adaptation («que tout change pour que rien ne change », selon le slogan de Lampedusa)?

Quant au GRET, l'association existe toujours (on fêtera ses 40 ans l'année prochaine), elle a même grossi et conquis une certaine notoriété. Une longévité surprenante quand on pense aux débuts, mais très satisfaisante et forcément significative d'une certaine pertinence du projet. Il n'y a d'ailleurs aucun problème de recrutement, des jeunes bien formés et motivés viennent y travailler volontiers en acceptant des salaires modestes et des rythmes de travail soutenus. Dans certains pays (Madagascar, Haïti, Cambodge, Mauritanie, Sénégal, etc.), le travail de coopération se mène depuis des années qui finissent par se compter en dizaines, avec des relations partenariales, perfectibles, mais certaines.

On ne parle plus de technologies appropriées, ni même d'appropriation, bon nombre de collègues ne connaissent d'ailleurs pas l'histoire du groupe. Maintenant, comme tout le monde, notre objectif est de réduire la pauvreté et les inégalités à travers les opérations que nous menons dans une vingtaine de pays, pour le compte principalement de la coopération européenne et de la coopération française. Il faut d'abord gagner les appels d'offre et ensuite gérer très rigoureusement les opérations, les procédures des bailleurs de fonds sont devenues très pointilleuses et grandes consommatrices de staff administratif. En effet, le risque de clôturer une opération de trois ans avec un solde négatif est très sérieux et le modèle économique est fragile. Dans quel sens le faire évoluer ? Le GRET est devenu une ONG, certains disent internationale parce que nous avons des antennes dans une dizaine de pays où nous salarions plusieurs centaines de personnes au total, mais l'argent continue de venir d'Europe et la direction reste centralisée à Paris. Peut-on dire franco-international ? On veut toujours influencer les politiques publiques mais les liens avec les ministères français ne sont plus ce qu'ils étaient et les pays partenaires revendiquent légitimement leur souveraineté. La coopération est-elle une ingérence ? Elle fait partie du « soft power » et des logiques d'influence, mais elle met aussi en œuvre des valeurs de solidarité. Dans le monde fortement globalisé d'aujourd'hui, dominé par le système financier, avec une autre géopolitique que la dichotomie Nord/Sud, une multiplicité d'acteurs (fondations, entreprises, ONG, etc.) venus contester le monopole des États sur les politiques sociales, des « externalités » (inégalités, réchauffement climatique, etc.) reconnus comme des paramètres inspirateurs de nouveaux modèles, la coopération au développement a vu son importance se relativiser sérieusement et en même temps rester d'actualité si elle sait opérer les mutations nécessaires. Les Objectifs du Développement Durable et les négociations en cours pour réduire le réchauffement climatique en sont la démonstration. Y compris dans son aspect technologique qui redevient, par le besoin d'innovation et les enjeux concurrentiels, un paramètre de ces Objectifs de Développement Durable. Même si l'économie politique, avec quelques prix Nobel à la clé (North, Orstrom), a consolidé le rôle déterminant des institutions. 
De ce point de vue, l'expérience du GRET est significative. Les méthodes et les solutions mises en œuvre combinent le technologique et l'institutionnel. Mais plus on est dans l'institutionnel plus on est intrusif surtout quand il s'agit de réduire les inégalités. Alors, la coopération solidaire, qui se veut partenariale et négociée, prend tout son sens, mais cela reste un chantier.

Donc globalement nous faisons face à de nouvelles et sérieuses mutations. De ce fait, il y a toujours besoin de prospective et bien ancrée dans l'Histoire. À quoi bon peut-être ? Comme le dit Yves Stourdzé ${ }^{13}$ : « toutes mémoires semblent définitivement perdues. (...) Les temps se troublent et les trajectoires se dissocient (...)». Mais justement, il ajoute : "Alors débutent d'extravagants recoupements, se fixent d'étonnantes relations transversales ». Toutes nos idées ne sont pas en ruines, elles font leur chemin.

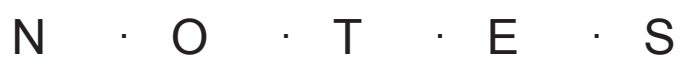

1. Il n'y avait pas, à l'époque, de ministère de la Recherche, seulement un secrétariat d'État.

2. Cf. : D. Sadoulet, La coopération au développement en France (1997-2004). Réforme et modernisation de l'État. L'Harmattan, 2007. Julien Meimon, «En quête de légitimité. Le ministère de la Coopération (1959-199) ». Thèse de doctorat en science politique, décembre 2005.

3. Thématique du Sommet de Versailles en 1982

4. Dans les années 1980, il y avait 20000 coopérants, pas tous techniques, il y avait de nombreux enseignants. Aujourd'hui il y en a un millier.

5. Stimulée par les approches globales, systémiques et historiques de professeurs comme Michel Sébillotte et Marcel Mazoyer, successeurs de René Dumont.

6. Pour plus d'information sur cette évolution lire : «Des jardins d'essai au Cirad : une épopée scientifique française ». Serge Volper et Hervé Bichat in Histoire de la recherche contemporaine, 2014 Tome III n².

7. Le CGIAR ou GCRAI en français (Groupe consultatif pour la recherche agricole internationale).

8. Il y aurait évidemment beaucoup plus à dire sur le rôle de la Banque mondiale, mais cela dépasse le propos de cet article.

9. Respectivement pour leur rendre hommage, Hervé Bichat, René Billiaz et Jacques Poly.

10. La réduction de moitié de l'extrême pauvreté à l'horizon de 2015 est devenue en 2000 le premier des Objectifs Millénaires du Développement (OMD)

11. Qui viennent d'être adoptés pour la période 20152030 par les Nations Unies pour faire suite aux OMD.

12. Christine Lagarde à France 2 en février 2015 et Angel Gurria le 22/10/15.

13. Y. Stourdzé, Les ruines du futur. Sens \& Tonka 11/24. 1979-1998. 


\title{
$R \cdot E ́ \cdot S \cdot U \cdot M \cdot E$
}

À la même époque que le CESTA une organisation dénommée GRET était créée pour tenter d'introduire de l'innovation dans les méthodes et les pratiques de la coopération avec les pays en développement. Un lien d'amitié entre les deux directeurs a permis d'opérer un certain dialogue entre les deux aventures. Celle du GRET permet de balayer le jeu des acteurs institutionnels concernés par cette politique de coopération et de témoigner sur des avancées et des difficultés à rapprocher de celles du CESTA.

\begin{abstract}
In the same period as CESTA existed, an organization called GRET was set up to try to introduce new methods and practices in cooperative relations with developing countries. The friendship between the directors of the two bodies made a certain amount of dialogue between them possible. Looking at GRET's history allows us insight into how institutional actors engaged in this cooperative policy performed, and to compare the successes and problems GRET experienced relative to those of CESTA.
\end{abstract}

